\title{
Research implications for teaching and learning strategies in undergraduate mathematics
}

\author{
Medhat Hishmat Rahim \\ Lakehead University, Thunder Bay, Ontario, Canada \\ For correspondence: mhrahim@lakeheadu.ca
}

\begin{abstract}
In preparing an address that fits well within the themes of the Frontiers in Mathematics and Science Education Research Conference 2014, I have chosen geometry. Evidently, geometric applications in Calculus, Algebra and other fields are essential for these disciplines' development. In addition, the widespread use of geometry by preservice and in-service teachers in elementary, middle, and secondary schools and universities makes it an indispensable discipline for a large population in education. In what follows I will try to highlight and present the main trends in recent research activities in teaching and learning undergraduate geometry that were carried out throughout the second half of the twentieth century up to the present. In particular, research on studying children and young adults' processes of how they construct geometric and spatial ideas of objects will be discussed. Additionally, research in mathematics on proofs and proving with selected examples of proving the Pythagorean Theorem visually and through geometric construction including my own proof will be introduced.
\end{abstract}

Keywords: construct, spatial, geometry, mathematics, calculus, proofs.

\section{Introduction and background}

Throughout the last half of the twentieth century, there have been critical didactic events in the area of thoughts development in geometry. Primarily, in the late 1950s, the van Hieles Model of Levels of Geometric Thoughts Development was introduced by Pierre van Hiele and Dina-Geldof van Hiele (Pierre van Hiele, 1984a, 1984b \& Dina van Hiele-Geldof, 1984a, 1984b). Initially the van Hieles model appeared within the writings of Dina van Hiele-Geldof (1984a) and Pierre van Hiele (1984b). The van Hieles model would seem to be one of the significant events in mathematics education. Consequently, the work and research of scholars in geometry education from the 1950s to the present have been energized worldwide with specific interest into the levels of students' geometric thinking processes and the methods of school teaching of geometry in the classrooms. In this review, the new studentcentered work and research activities in geometry may be classified into two main categories: Research in Thought Development in Geometry, and Research in Methods of Teaching Geometry. The following is an attempt to introduce major events in each of these categories and the influences of the two trends on each other as the allocated space allows.

\section{Research in Thought Development in Geometry}

A well-known research work in this category has been the establishment of a research based model of Levels of Thought Development in Geometry known as the van Hieles Levels of the Development of Geometric Thinking introduced by Dina-Geldof van Hiele and Pierre van Hiele in the 1950s (Shaughnessy and Burger, 1985). The impact the van Hieles model has made on the geometry curricula and its teaching methods along with a number of other related research projects will be discussed.

The van Hieles Levels of Thought Development in Geometry (1950s). Van Hieles research have come up with five levels of geometric thinking development that the student would progress through subject to the geometry instruction he/she receives regardless of his/her biological growth. That is, a student may remain at the first level of geometric thinking for the rest of his/her life if no instruction is made to 
progress the student to the next level. In other words, student's progress from a level to the next does depend on instruction and content received. No method of instruction permits the learner to skip a level. In contrase, Piaget's cognitive development levels [Sensorimotor (0-2 yrs), Pre-operational (2 - 6 yrs), Concrete-operational (6-12 yrs), Formal-operational (12 -16 yrs)] are due to biological growth and progress from level to the next is automatic (Piaget, Inhelder, \& Szeminska, 1960).

The van Hieles five levels were described in the literature as follows: Level 0: Visualization; Level 1: Analysis; Level 2: Informal Deduction; Level 3: Formal Deduction; and Level 4: Rigor; and outlines of these levels, based on Crawley (1987) and Shaughnessy \& Burger (1985), are given below:

\section{Level 0: Visualization}

Students at this level are aware of their surrounding as something that exists around them. Geometric objects are viewed in their totality with no awareness to their components or attributes; that is, geometric objects or figures are identified by their shape as a whole ... by their physical looking and not by their parts or properties. For example, a person at this level can learn geometric vocabulary, can identify specified shapes like a square or a rectangle and can reproduce a given square or rectangle. However, a person at this level would not recognize that the square or rectangle has right angles or that the opposite sides are parallel.

\section{Level 1: Analysis}

In this level students begin analysing geometric concepts through observation and experimentation where they start to distinguish and recognized properties of figures; and the recognized properties are utilized to identify classes of shapes. As such, figures are identified by their parts. For example, for a given parallelogram, students can, by marking the equal angles, conclude that the opposite angles in parallelograms are equal. After experimenting of several parallelograms, students can make generalization for the class of parallelograms. Interrelationships among figures and among properties are not reached, and definitions are not realized.

\section{Level 2: Informal Deduction}

Students at this level can establish interrelationships of properties within a given figure (e.g., in a triangle, the three sides being equal necessitate its three angles being equal). Also, students at this level can establish interrelationships among figures (e.g., a square is a parallelogram because its opposite sides are parallel too). Therefore, students at this level can figure out properties of a figure and identify classes of figures; and, class inclusion as well as definitions, are realized. However, students at this level do not realize the important role of axioms, formal proofs, and the significance of deductive reasoning.

\section{Level 3: Formal Deduction}

The importance of deduction as a method to create a theory through certain axioms is realized at this level. The role of axioms, definitions, theorems, undefined terms, postulates, and proofs are understood. The students at this level can establish proofs; they realize that developing a proof can be done in different ways.

\section{Level 4: Rigor}

In this level students can function in a number of axiomatic systems; they can study nonEuclidean geometries. Geometry, therefore, is approached in the abstract. (Shaughnessy and Burger, 1985, p. 420, and Crowley, M., 1987, pp. 2-3).

Wirszup's Report (1976): Breakthroughs in the Psychology of Learning and Teaching Geometry. Historically, the van Hieles model was introduced in the late 1950s through the writings of Dina van Hiele-Geldof (1984a, 1984b) and Pierre van Hiele (1984a, 1984b). However, although the model was introduced in 
the late 1950s, it was not until the 1970s the model started getting publicity around the world through scholars' writings, presentations, and publications such as Freudenthal (1973) and Issak Wirszup (1976). In this regard, it is interesting to recall what Wirszup (1976) has once commented on the problematic status of undergraduate geometry in USA in which he referred extensively to the van Hieles levels of geometric thought development. In his words, Wirszup (1976) concluded that,

\begin{abstract}
A review of the teaching of geometry in the United States indicates at once that only a very small number of the elementary schools offer any organized studies in visual geometry, and where they are done, they begin with measurements and other concepts which correspond to Levels II and III of thought development in geometry. Since Level I is passed over, the material that is taught even in these schools does not promote any deeper understanding and soon completely forgotten. Then, in the $10^{\text {th }}$ grade, 15 and 16 year old youngsters are confronted with geometry for almost the first time in their lives. The whole unknown and complex world of plane and space is given to them in a passive axiomatic or pseudo-axiomatic treatment. The majority of our high school students are at the first level of development while the course they take demands the fourth level of thought. It is no wonder that high school graduates have hardly any knowledge of geometry, and that this irreparable deficiency haunts them continually later on (p. 96)
\end{abstract}

Piaget's Position on Learning and Teaching Geometry. As a matter of fact, the problematic status of undergraduate geometry outlined above by Wirszup (1976), was recognized much earlier by Piaget (1962). In making an observation on the learning and teaching geometry while commenting on Vygotsky's critical remarks concerning The Language and Thought of the Child, Piaget (1962) stated that,

... it begins late, ... it is specifically geometrical or even metrical without first going through a qualitative phase in which spatial operations would be reduced to logical operations, applied to a continuum ... it should be clear that to my mind it is not the child that should be blamed for the eventual conflicts, but the school, unaware as it is of the use it could make of the child's spontaneous development, which it should reinforce by adequate methods instead of inhibiting it as it often does (pp. 7-8).

The preceding views on the state of affairs in school geometry clearly indicate its problematic nature where complex concepts (measurement aspects of shapes) are being used to understand simpler concepts (visual properties of shapes).

\title{
Research in Methods of Teaching Geometry
}

Since Wirszup's assessment (1976), school geometry has witnessed a slow improvement and little change. The major contributions remain the work of the van Hieles and its impact on East European scholars. At the mid 1970s, the East European countries, have initiated overall revisions of the school geometry curricula at all school levels based on the van Hieles levels of geometric thinking (Wirszup, 1976). Furthermore, in North America, and by the mid 1970s, gradual and steady efforts for improvement of what and how to teach undergraduate geometry have taken place. In particular, the National Council of Teachers of Mathematics (NCTM) has introduced its document, Curriculum and Evaluation Standards for School Mathematics (1989), based on the new research findings throughout the world. Consequently, more scholars have adopted van Hieles model. Thus, a number of proposed investigative works in visual literacy were initiated and published in the form of new approaches documents, texts, booklets, and teaching materials. The following is a review of a sample of some exemplary products in the forms of texts, research projects and position papers in learning and teaching geometry along the van Hieles levels.

\section{Text Books}

Geometry: An Investigative Approach, by Phares G. O'Daffer \& Stanley R. Clemens (1976, 1 ${ }^{\text {st }}$ Edition \& 1992, $2^{\text {nd }}$ Edition). 
O'Daffer \& Clemens (1992) have made it clear that their text was designed along the research findings of van Hieles development levels of geometric thinking. In particular, O'Daffer \& Clemens have made the following assertions along the new movement of applying the van Hieles levels throughout the text,

... Each chapter also contains numerous Investigation sections. These activities are developed and sequenced according the Van Hiele levels for learning geometry: Level 0 Visualization; Level 1: Analysis; Level 2: Informal Deduction; Level 3: Deduction; Level 4: Rigor. The primary emphasis in this text is on Levels 0 through 2, which include the following ability goals:

Visualize geometric figures: manipulate (color, cut out, fold and so on) geometric figures. Classify geometric figures: Sort figures according to selected attributes, compare and contrast figures; ..., develop and use a definition for a figure; describe inclusion relationships for figures.

Analyze geometric figures/relationships: Explain how figures and parts of figures are related; interpret the meaning of a geometric situation.

Discover/formulate generalizations: Answer and pose "what if" questions; ...; discover generalizations from empirical consideration of examples.

"Proving" and disproving generalizations: Give simple reasons to convince someone that something is true; present informal, convincing arguments that something is true (using cut-outs, measurement, construction, diagrams, computers, and so on); follow informal arguments that show that something is true; show a counterexample for a false generalization (pp. vii-viii).

The text, O'Daffer \& Clemens (1992), covers fundamental aspects in geometry throughout ten chapters. For example, Chapter 1 covers Geometry in the physical world, Geometry as a Mathematical System, Geometry as a Formal Axiomatic Structure, and Aesthetic and Recreational Aspects of Geometry together with Pedagogy for Teachers; Chapter 2 focuses at Basic Ideas of Geometry while Chapter 3 covers Discovering Polygonal Relationships and Chapter 4 deals with Patterns of Polygons through Tessellations ... As a note, I have used this text for several years in a geometry course run at the mathematics department, University of Alberta, Canada and found the students so excited with the text.

\section{Research Projects on Teaching Geometry}

Children Exploration of shapes through cut and cover: Learning from Rearranging Shape. Medhat H. Rahim, Daiyo Sawada and Johnna Strasser's Project (1996).

An action research project in the classroom, titled Children Learning from Rearranging Shapes was conducted with grade 4 students (Rahim, Sawada, and Strasser, 1996). The following is a brief reflection on the project.

Miss Strasser has taken the teaching role for a grade 4 class in the state of Wisconsin, USA. The project was designed along van Hieles Levels of geometric thought development and guided by the recommendations of the National Council of Teachers of Mathematics (NCTM) Standards for School Mathematics (1989), USA. The NCTM Standards recommend,

In grades K-4, NCTM recommended changes to the content and emphasis of the mathematics being taught. Decrease attention to be placed on the rote use of symbols and operations, and increased attention placed on number sense, estimation, and reasoning ... Increased attention was to be placed on geometry and measurement, extending beyond the naming of geometric figures and converting between units of measure. New topics would include the properties of geometric figures and relationships between them, developing spatial sense, concepts related to measurement and 
estimation of measure. (Link below), http://www.mathcurriculumcenter.org/PDFS/CCM/summaries/standards summary.pdf

Along these recommendations, the research team has produced a set of simple geometric materials intended to assist student exploration of shapes undergoing transformation from one shape (e.g. a triangle) into another (say a rectangle). Throughout such transformation processes, a main attribute of shape, the area, remains invariant. The materials used consisted of a set of full sized copies of each of the 6 shapes shown in Figure 1 and scissors. These shapes were provided, one at a time, to each student throughout classroom teaching. Figure 1 below depicts the first sheet, labelled as the template, and the other five shapes labelled A to E. The two pieces of the template are to be cut out and used by children to construct the other shapes A to E. The horizontal line defining the two pieces of the template joins the midpoints of the two sides of the triangle. The teacher prepared overhead transparencies of the template and the rest of the shapes for demonstration uses.

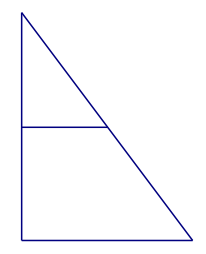

Template

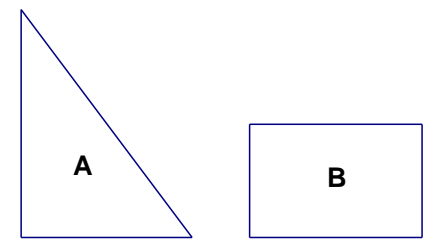

Figure 1. The six shapes used in the classroom investigative project

\section{The Exploration}

The scenario summarized here is reconstructed from several experiences with grade 4 class of 24 students dealing with shape-to-shape, shape-to-part and part-to-part relationships. In the first classroom session, the joyful teacher, Johnna Strasser, has asked the children to assume they are 'engineers' in the 'land of shapes' and herself as 'the chief engineer'. By using the two pieces of the template, engineers were instructed by their chief to build each of the five shapes A to E one at a time. First, the teacher provided each student with a full size copy of the template then asked each student to cut his/her template into its two pieces and label the upper piece 1 and the lower piece 2 . The teacher performed the cutting and labelling using the transparency while students worked with paper handouts at their desks. Also, full size copies for each shape were supplied one at a time as the teaching progresses. The students were informed that by sliding, flipping, and/or turning pieces 1 and 2 they can construct the shape shown on the supplied activity sheet. They were asked to name each of their construction products.

\section{Classroom Observations of Students Work}

It was an easy task for all students to cut the template along the dissection line into two pieces and label them 1 and 2. And, cutting the template into two pieces and building another triangle (triangle A) was so simple for the whole class. This exploration provided an introduction practice for the students to the nature of the decomposition-composition process by cutting then sliding, flipping and/or turning the pieces. All students did correctly name their construction result as a triangle. However, for Shape B, the rectangle, two children have some difficulties with constructing the rectangle (shape B). Instead they tried to compose and build the shape B on their desk using the activity sheet as visual guide rather than attempting to cover it by the two pieces 1 and 2 . They came up with the shape shown below insisting the shape is a rectangle. 


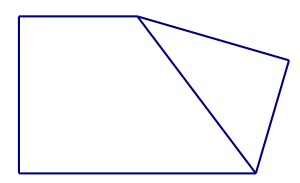

To this end, the teacher interrupted the class to ask for familiar objects that have the shape B. Children responded: a door, a book, the blackboard, a window. The two children tried further sliding, turning and flipping to get eventually the rectangle. 13 children named the shape as a rectangle and 11 called it a square. Interestingly, many children showed doubt that the rectangle could be built. Clearly, this is a signal that these children did not have a qualitative experience with shapes. At this point the teacher decided to review progress. Manipulating the two transparency pieces, she composed shape A (triangle) and B (rectangle) on the overhead projector. She then asked: whether or not the two shapes A and B were different in shape; and whether or not they differed in size. While all students agreed that the objects had different shapes, they disagreed about whether or not they covered the same amount of space. Below is a sample of some children's answers and reasoning:

No, they are not the same sizes: because they have different number of sides; because they have different lengths of sides; because shape $A$ is taller it is larger.

One boy in the class took out his ruler and measured all the sides of shapes A and B, summed up his measures for each, and declared that shape A was $6 \mathrm{~cm}$ bigger than shape $\mathrm{B}$.

Other children responses:

Yes, they are the same size: because they weigh the same; because they have the same amount; because they could hold the same stuff; because they are the same pieces just moved around; because they are the same shape just cut in half.

These conflicts concerning the size question made the teacher to clarify that by size it is meant the amount of space inside the shape; she stressed that moving a piece around does not change its size. Nonetheless, 'the boy with the ruler' was not impressed. The uncertainty of the size of a shape being invariant under the decomposition-composition process would be due to the lack of spatial experience and practice in schools. In this regards, Rahim and Sawada (1990) have suggested earlier that shape transforms through cut and cover can provide a qualitative basis for understanding geometric relationships among shapes. In their reflection on the early geometry curricula in schools, they stated,

In the early grades, there are some 'play activities' with the basic polygonal shapes leading to the recognition, reproduction, and labelling of squares, triangles, etc. Very little else is done of a qualitative nature. Instead the emphasis is very soon on determining and using metric properties of polygons such as perimeter and area (these are the 'measurement' foci that Wirszup, 1976, refers to), (p. 303).

Evidently, 'the boy with the ruler' is a case in point here. He has been left high and dry! He could not appreciate nor understand that the processes of sliding, turning or flipping of a shape leave the area of the shape unchanged! 


\section{Research in Proofs and Proving: the case of the Pythagorean Theorem}

A close look in the literature would immediately show that Loomis' text (1968), The Pythagorean Proposition, would be a distict resource for the ongoing efforts of proofs and proving applied to the Pythagorean Theorem. Loomis' text presents 370 proofs of the theorem. Loomis have detailed these proofs included in his text as follows,

On May 1, 1940 at the revised completion of the manuscript for my $2^{\text {nd }}$ edition of The Pythagorean Proposition, it contains - - proofs: Algebraic, 109; Geometric, 255; Quaternionic, 4; Dynamic, 2; in all 370 different proofs, each proof calling for its own specific figure. And the end is not yet (p. 269).

The literature further suggest another collection of proofs for the Pythagorean Theorem prepared by Bogomolny (1996-2012), known as Bogomolny's collection compiled and made available through the Internet by Bogomolny's internet link, http://www.cut-the-knot.com/pythagoras/ . This collection presents 101 different proofs of the Pythagorean Theorem, some of which were presented earlier in Loomis' text and the rest are presumably new proofs. As the above citation suggests, Loomis' text does not contain a Trigonometric proof for the theorem but Bogomolny's collection does, namely the $84^{\text {th }}$ proof, within the 101 proofs he provided. In this regard, Bogomolny states that,

I must admit that, concerning the existence of a trigonometric proof, I have been siding with Elisha Loomis until very recently, i.e., until I was informed of Proof \#84. Actually, for some people it came as a surprise that anybody could doubt the existence of trigonometric proofs, so more of them have eventually found their way to these pages (Bogomolny's internet link http://www.cut-the-knot.com/pythagoras/).

The trigonometric proof of the Pythagorean Theorem was published by Jason Zimba (2009). To view Zimba's proof, just click at the link Proof \#84 and follow instructions.

\section{Proof of the Pythagorean Theorem through Construction (Rahim, 2003)}

To show that,

For two squares of $m$ and $n$ units, there can be constructed a third square of $x$ units of length using a compass and unmarked straight edge such that $m^{2}+n^{2}=x^{2}$.

Rahim's (2003) summary: Consider two squares ABCD and EFGH shown below.

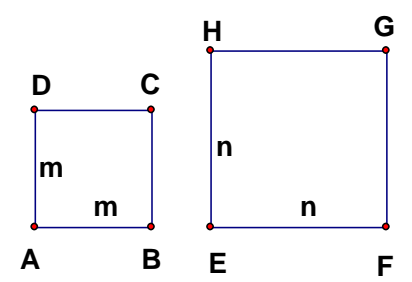

Then, three constructions shown in Figure 1 below are required for the proof: (a) Construction of rectangle BKJI with area $=$ area of square $A B C D$ with $B I=n$; (b) Construction of rectangle BSQI with area = area of $\mathrm{ABCD}+$ area of $\mathrm{EFGH}$; (c) Construction of $\mathrm{BT}=\mathrm{x}$ units as the length for the required third square that would satisfy $m^{2}+n^{2}=x^{2}$. 


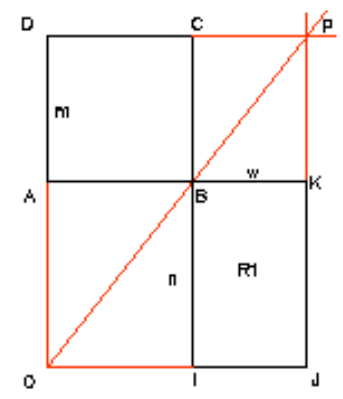

(a) (b)

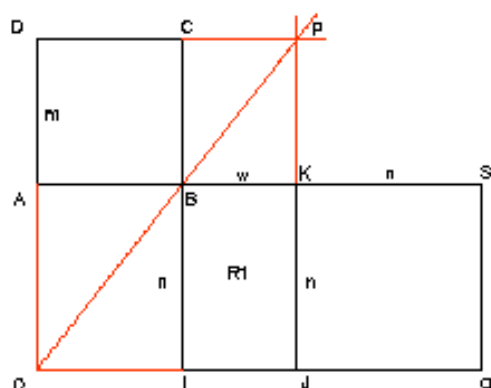

(c)

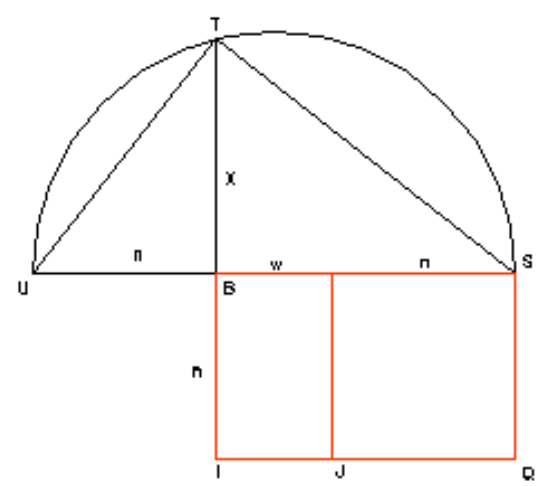

(b) BSQI = Square ABCD + Square EFGH,

Figure $2^{*}$. Constructions: (a) BKJI = square ABCD;

\section{(c) BT $=x$ units}

* (Figure 1 consists of replicas of Rahim's original figures. The construction steps for the required square of $x$ units that satisfies $m^{2}+n^{2}=x^{2}$ have been presented with animations though a power point document available through the Internet link http://mrahim.lakeheadu.cal.)

A brief description for the three constructions:

(a) Constructing a rectangle with area equals $\mathrm{m}^{2}$.

As shown in Figure 2(a), the area of rectangle BIJK (or $\mathrm{R}_{1}$ ) = area of the square ABCD; it is easy to follow; thus $\mathrm{nw}=\mathrm{m}^{2}$.

(b) Constructing a rectangle with area equals the combined areas of the two squares.

Since $\mathrm{BI}=\mathrm{KJ}=n$ units, align the square EFGH along the line segment $\mathrm{KJ}$ as shown in Figure $2 \mathrm{~b}$, and the required rectangle BIQS is constructed.

Thus, area BIQS $=$ rectangle BKJI + square $\mathrm{EFGH}=\mathrm{nw}+\mathrm{n}^{2}=m^{2}+\mathrm{n}^{2}$.

(c) Constructing a square with area equals the area of the rectangle BIQS.

Figure 2c shows that $\Delta$ UTS is a right triangle (drawn in a half circle) of

diameter $=(n+w+n)$ units and height $T B=x$ units. And, the right $\Delta \Delta$ SBT and UBT are similar (since corresponding angles are equal), thus corresponding sides are proportional, that is,

$\mathrm{TB} / \mathrm{UB}=\mathrm{SB} / \mathrm{TB}$ implies

$(\mathrm{TB})^{2}=(\mathrm{UB})(\mathrm{SB})$, then with $\mathrm{TB}=\mathrm{x}$ units, it follows that $x^{2}=n(w+n)=n w+n^{2}=m^{2}+n^{2}$. (Rahim, 2003, pp. 146-149).

Note that, (1) it is felt necessary to skim Rahim's (2003) proof so that the reader would have an immediate access to the heart of the proving procedure; (2) the use of similarity of $\Delta \Delta$ SBT \& UBT was NOT used in Rahim's (2003) original proof. The original proof takes TB as the geometric mean for UB and $\mathrm{BS}$ within the right $\Delta \mathrm{UTS}$ resulting to have $\mathrm{TB} / \mathrm{UB}=\mathrm{BS} / \mathrm{TB}$ etc.

\section{Epilogue}

In this article an attempt has been made to introduce a review for research in undergraduate geometry of the child geometric thinking development, and methods of teaching geometry together with a trace on activities in proofs and proving with reference to Pythagorean Theorem. Additionally, piece-wise congruence of area equivalent shapes (or congruence by pieces) through decompositioncomposition (dissection-motion) operations was introduced and reported particularly as grade 4 children were observed attempting these shapes transformations in the classroom. In doing so, a generalization of the congruence concept to piece-wise congruence was made where the 'same shape' condition was dropped. The crucial point here is that congruence implies area equivalence but the 
reverse is not necessarily true whereas piece-wise congruence implies area equivalence and the reverse is true too. Eves, (1972) expresses piecewise congruence idea as follows, "The two polygons can thus be considered as made up of sets of corresponding pieces which are congruent in pairs but perhaps fitted together differently" (p. 194).

\section{References}

Bogomolny, A. (2012). A collection of 101 proofs for the Pythagorean Theorem compiled in the Internet Link: http://www.cutthe-knot.com/pythagoras/

Commission on Standards for school Mathematics (1989). Curriculum and Evaluation Standards Report. The internet link: http://www.mathcurriculumcenter.org/PDFS/CCM/summaries/standards_summary.pdf

Crawley, Mary L. (1987). The van Hiele Model of the Development of Geometric Thought. In Learning and Teaching Geometry, K12, 1987 Yearbook of the National Council of Teachers of Mathematics, edited by Mary M. Lindquist, pp. 1-16. Reston, Va.: NCTM.

Eves, H. (1972). A Survey of Geometry. Boston: Allen \& Bacon.

Freudenthal, Hans. (1973). Mathematics as an Educational Task. Dordrecht, Netherland: D. Reidel.

Loomis, E. S. (1968). The Pythagorean Proposition. Reston, VA: NCTM.

National Council of Teachers of Mathematics (1989). Curriculum and Evaluation Standards for School Mathematics. Rosten, VA.: NCTM.

O’Daffer, P., \& Clemens, S. (1992). Geometry: An Investigative Approach. Addison-Wesley Publishing Co.

Piaget, J. (1962). Comments on Vygotsky's critical remarks concerning The Language and Thought of the Child, and Judgment and Reasoning in the Child. The MIT Press, Massachusetts Institute of Technology (MIT).

Piaget, J., Bärbel Inhelder, and Alina Szeminska (1960). The Child's Conception of Geometry. New York: Basic Books, Inc., 1960.

Rahim, M. (2003). A new proof of the Pythgorean using a compass and unmarked straight edge. International Journal of Mathematical Education in Science and Technology, Vol. (34), No. 1, January-February, 144-150.

Rahim, M, Sawada, D., \& Strasser, J. (1996). The Boy with the ruler. The Mathematics Teaching (MT),Vol. 154, March 1996, pp. 2329.

Rahim, M., \& Sawada, D. (1990). The Duality of qualitative and quantitative knowing in school geometry. International Journal of Mathematical Education in Science and Technology, Vol. (21), No. 2, 303-308.

Shaughnessy, J.M., \& Burger, W.F. (1985). Spadework prior to deduction in geometry. Mathematics Teacher, 78, 419-428.

van Hiele, Pierre M. "A Child's Thought and Geometry." In English Translation of Selected Writings of Dina van Hiele-Gildof and Pierre M. van Hiele, edited by Dorothy Geddes, David Fuys, and Rosamond Tischler as part of the research project "An Investigation of the van Hiele Model of Thinking in Geometry among Adolescents," Research in Science Education (RISE) Program of the National Science Foundation, Grant No. SED 7920640. Washington, D.C.: NSF, 1984a. (Original work published in 1959.)

van Hiele, Pierre M. "English Summary by Pierre Marie van Hicle of the Problem of Insight in Connection with School Children's Insight into the Subject Matter of Geometry." In English Translation of Selected Writings of Dina van Hide-Geldof and Pierre M. van Hiele, edited by Dorothy Geddes, David Fuys, and Rosamond Tischler as part of the research project "An Investigation of the van Hicle Model of Thinking in Geometry among Adolescents," Research in Science Education (RISE) Program of the National Science Foundation, Grant No. SED 7920640. Washington, D.C.: NSF, 1984b. (Original work published in 1957.)

van Hiele-Geldof, Dina. "Dissertation of Dina van Hiele-Gcldof Entitled: The Didactic of Geometry in the Lowest Class of Secondary School." In English Translation of Selected Writings of Dina van Hiele-Geldof and Pierre M. van Hiele, edited by Dorothy Geddes, David Fuys, and Rosamond Tischler as part of the research project "An Investigation of the van Hiele Model of Thinking in Geometry among Adolescents," Research in Science Education (RISE) Program of the National Science Foundation, Grant No. SED 7920640. Washington, D.C.: NSF, 1984a. (Original work published in 1957.)

van Hiele-Geldof, Dina. "Last Article Written by Dina van Hiele-Geldof entitled: Didactics of Geometry as Learning Process for Adults." In English Translation of Selected Writings of Dina van Hiele-Geldof and Pierre M. van Hiele, edited by Dorothy Geddes, David Fuys, and Rosamond Tischler as part of the research project "An Investigation of the van Hiele Model of Thinking in Geometry among Adolescents," Research in Science Education (RISE) Program of the National Science Foundation, Grant No. SED 7920640. Washington, D.C.: NSF, 1984b. (Original work published in 1958.)

Wirszup, Izaak. (1976). Breakthroughs in the Psychology of Learning and Teaching Geometry. In Space and Geometry: Papers from a Research Workshop, edited by J. Martin. Columbus, Ohio: ERIC/SMEAC.

Zimba, J. (2009). On the Possibility of Trigonometric Proofs of the Pythagorean Theorem Forum Geometricorum, Volume 9, 275- 